\title{
Análisis de las motivaciones y de las expectativas de los estudiantes de máster en Educación
}

\author{
Pedro Jurado de los Santos \\ Universitat Autònoma de Barcelona. España. \\ pedro.jurado@uab.cat \\ Pilar Figuera Gazo \\ Juan Llanes Ordóñez \\ Universitat de Barcelona. España. \\ p.figuera@ub.edu \\ juanllanes@ub.edu
}

Recibido: 27/10/2017

Aceptado: 1/10/2018

Publicado: 15/7/2019

\section{Resumen}

Este trabajo explora la percepción del alumnado de másteres del ámbito de la educación con relación a variables de transición, satisfacción y perspectivas de futuro, poniendo el énfasis en el papel de las motivaciones y las expectativas. Mediante un procedimiento metodológico descriptivo, se ha aplicado un cuestionario a una muestra de 297 estudiantes de másteres en Educación. Los resultados indican lo siguiente: las motivaciones para acceder a ellos se relacionan prioritariamente con el hecho de ampliar el bagaje formativo, los niveles altos de satisfacción académica se asocian a la relación entre compañeros y la perspectiva profesional se orienta hacia la inserción laboral. Por otra parte, pueden asumirse las dificultades de los estudiantes para gestionar la carrera prospectivamente a partir del máster, así como la oportunidad que ofrece este para la especialización, el acceso al doctorado como finalidad y el ejercicio profesional dentro de los distintos proyectos personales. Se destacan perfiles heterogéneos de los alumnos condicionados por la experiencia profesional previa, lo que determina su posicionamiento ante los procesos formativos y sus expectativas con relación a los mismos.

Palabras clave: expectativas profesionales; transición a la vida profesional; motivación; satisfacción académica; máster universitario

\section{Resum. Anàlisi de les motivacions i de les expectatives dels estudiants de màster en Educació}

Aquest treball explora la percepció d'estudiants de màster en el camp de l'educació en relació amb variables relacionades amb la transició, la satisfacció i les perspectives de futur, posant l'èmfasi en el paper de les motivacions i les expectatives. Mitjançant un procediment metodològic descriptiu, s'ha aplicat un qüestionari a una mostra de 297 estudiants dels màsters en Educació. Els resultats indiquen que les motivacions per accedir-hi es relacionen principalment amb la intenció d'ampliar el seu bagatge formatiu, que els alts nivells de satisfacció acadèmica s'associen amb la relació amb els companys i que la perspectiva professional s'orienta cap a la inserció laboral. D'altra banda, es podem assumir les dificultats dels estudiants per gestionar la carrera de forma prospectiva a partir del màster, així com l'oportuni- 
tat que ofereix aquest per especialitzar-se, accedir al programa de doctorat com a finalitat i incloure l'exercici professional dins dels diferents projectes personals. S'hi destaquen perfils heterogenis dels alumnes condicionats per l'experiència professional prèvia; això en determina el posicionament davant dels processos formatius i de les expectatives que tenen en relació amb aquests.

Paraules clau: expectatives professionals; transició a la vida professional; motivació; satisfacció acadèmica; màster universitari

\title{
Abstract. Analysis of the motivations and expectations of master's students in education
}

This paper explores the perception of master's students in education related to transition, satisfaction, and perspectives with an emphasis on the role of motivations and expectations. Using a descriptive methodological procedure, a questionnaire was administered to a sample of 297 students enrolled in master's degree programs in education. The results indicate that students' motivations for entry are primarily related to expanding their educational background; high levels of satisfaction are associated with the relationship with colleagues; and the professional perspective is oriented towards labor insertion. In contrast, students encounter difficulties in managing their careers upon completion of the program, as well as opportunities for specialization, access to doctoral programs, and professional practice within various personal projects. The study reveals that the students' profiles are heterogeneous and conditioned by previous professional experience, which determines their attitude towards formative processes and their expectations in relation to them.

Keywords: professional expectations; transition to professional life; motivation; academic satisfaction; master's degree

\author{
Sumario \\ 1. Introducción 4. Conclusiones \\ 2. Método Referencias bibliográficas
}

3. Resultados

\section{Introducción}

En la última década, y como consecuencia de los cambios introducidos por la declaración de Bolonia (1999), se ha asistido a una reorganización de los estudios universitarios. La incorporación de los másteres como un espacio de desarrollo profesional ha sido uno de los cambios más significativos introducidos en la educación superior. De este modo, la implantación del EEES a nivel nacional ha supuesto una nueva estructura de las enseñanzas universitarias oficiales en tres nuevos ciclos: primer ciclo (grado), segundo ciclo (máster) y tercer ciclo (doctorado). Desde su implementación, la oferta de másteres se ha incrementado sustancialmente y la matrícula ha crecido en un $138 \%$ desde el año 2008 (MECD, 2015). Las diferencias son significativas por tipo de universidad (un $73 \%$ en la universidad pública) y rama de conocimiento (un $56 \%$ en Ciencias Sociales). La importancia cuantitativa y cualitativa de 
este nivel formativo se constata, también, en el contexto internacional (Hauschildt, Gwosć, Netz, y Mishra, 2015).

Tal y como define el Ministerio de Educación y Ciencia (RD 1393/2007, de 29 de octubre), los másteres oficiales tienen como finalidad la adquisición por parte del estudiante de una formación avanzada de carácter especializado o multidisciplinario, orientada a la especialización académica o profesional o a la investigación. La iniciación en tareas de investigación ha quedado reforzada con el Real Decreto 420/2015, art. 7, de 29 de mayo, al establecer que el 70\% deben ser doctores. En el contexto español, la formación de máster oscila entre 60 y 120 créditos ECTS, y comparten el objetivo de formar al estudiante en competencias profesionales de alto nivel, tal y como indican los descriptores de Dublín ratificados en la Conferencia de Ministros Europeos de Educación Superior que tuvo lugar en Bergen en mayo de 2005.

La información aportada por los diferentes mandatos comunitarios señala la relevancia del aprendizaje a lo largo de la vida, como un instrumento para garantizar a la ciudadanía la actualización de las competencias (Informe PIAAC, 2013; Council of the European Union, 2011). La crisis económica que sufren muchos países de nuestro entorno europeo ha contribuido a incrementar el número de profesionales que retornan a la formación tras años fuera del sistema universitario, con la finalidad de incrementar su empleabilidad (OCDE, 2015). Estos demandantes se suman a los recién graduados que prolongan la formación ante la falta de perspectivas profesionales y amplían sus años de estudio, esperando con ello estar mejor preparados para situarse en la complejidad del nuevo paradigma del mercado de trabajo (Savickas, 2012; Savickas et al., 2009).

Las rápidas transformaciones ocurridas en el contexto mundial se ven reflejadas en los ámbitos familiares, educativos y laborales, y exigen nuevos retos a los estudiantes y también a las instituciones educativas, quienes deben preparar a los ciudadanos para afrontar situaciones complejas en sus sucesivas transiciones laborales. En este sentido, es fundamental conocer a los estudiantes de máster para contribuir a su orientación académica y profesional.

El análisis del perfil del estudiante de máster confirma una tendencia a la diversificación en lo que respecta a la procedencia formativa y social. Entre las causas, señalamos los cambios en las políticas de empleabilidad y formación a lo largo de la vida (Romero y Figuera, 2016), el logro de los principios de equidad derivados de la dimensión social (Egido-Gálvez, Fernández-Díaz y Galán, 2014) y los retos de la internacionalización de los sistemas universitarios (Rienties, Luchoomun y Tempelaar, 2014). La atracción internacional de las enseñanzas es muy superior en los estudios de máster oficial, con una presencia de estudiantes extranjeros del $11,4 \%$ frente al 1,7\% en los grados. Se constatan diferencias en función de los másteres y del ámbito académico (Armenteros y Pérez, 2015). Paralelamente, la información muestra un aumento del abandono, cuya tasa a nivel nacional es del $22,9 \%$, un $15 \%$ del cual corresponde al abandono en el primer año. Los datos varían en función del tipo de universidad y área de estudio (MECD, 2015). 
A partir de las escasas investigaciones sobre las transiciones en los másteres, destaca en nuestro país de manera significativa la línea de investigación relacionada con el máster de formación inicial del profesorado de secundaria, en la que se analizan expectativas, concepciones previas, construcción de la identidad e integración (Hernández y Carrasco, 2012; Manso y Martín, 2014; Serrano y Pontes, 2015). En otro tipo de másteres, sin embargo, estos análisis constituyen líneas aún emergentes. Destacan, también, los trabajos de LlanesOrdónez, Figuera-Gazo, Jurado-De los Santos, Romero-Rodríguez y TorradoFonseca (2015), puesto que aportan una visión comparada del perfil y de los procesos de transición en los másteres del área de educación en el contexto iberoamericano, donde se analizan tres tipologías de estudiantes: aquellos que acceden a un máster directamente desde un primer ciclo universitario y/o sin experiencia laboral, quienes lo hacen con una experiencia profesional inferior a cinco años (representados como júnior) y profesionales con una experiencia laboral más consolidada (conocidos como sénior). La procedencia del estudiante marca las expectativas que se derivan de su formación, así como la satisfacción académica respecto a sus estudios (Figuera, Buxarrais, Llanes y Venceslao, 2018; Pegalajar, 2016; Soilemetzidis, Bennett y Leman, 2014). Ello determina que los motivos intrínsecos de acceso vinculan al alumno de manera positiva con la formación.

A pesar del incremento de la población universitaria de máster, se confirma la ausencia de un corpus de investigación amplio sobre las transiciones en este nivel (Cohen, 2012). La procedencia investigadora remite a una amplia bibliografía sobre los estudios de grado (véase la revisión de Cabrera, Pérez y López, 2014) y también encontramos diversos trabajos de investigación sobre transición de estudiantes de doctorado, fundamentalmente en el contexto anglosajón (Rapp y Golde, 2008; Ramírez, 2014). En diversos países se han iniciado proyectos en esta línea que persiguen profundizar en las características del proceso de transición, la satisfacción, las dificultades de los estudiantes y los momentos críticos. Entre los programas de más amplio alcance destacamos la encuesta anual sobre la experiencia académica de estudiantes de postgrado realizada por The Higher Education Academic (UK), que analiza las motivaciones y las expectativas en el acceso, la satisfacción con la experiencia académica y los factores personales e institucionales que han condicionado su valoración (Soilemetzidis et al., 2014), y el proyecto Master's Completion, del Council of Graduate School (USA), basado en un análisis amplio sobre la adaptación y la persistencia en los estudios de máster (Barry y Mathies, 2011). $\mathrm{O}$, en el caso del territorio catalán, la encuesta diseñada por la Agencia para la Calidad del Sistema Universitario Catalán, donde se indaga acerca de la inserción laboral de los estudiantes de segundo ciclo (AQU, 2015).

En este trabajo se presentan los resultados de una investigación desarrollada con alumnos de másteres en el ámbito de la educación y ubicada en el entorno de las Ciencias Sociales, cuyos estudios han asistido a un proceso de transformación muy importante, con una diversificación de titulaciones en los niveles de grado y de másteres. Las cuestiones que se plantean son: ¿Cuál es el perfil de 
los universitarios que acceden a los estudios de máster en el área de educación? ¿Qué diferencias se presentan entre los estudiantes en función de su trayectoria laboral previa? ¿Cuáles son sus trayectorias laborales? ¿Qué nivel de satisfacción muestran respecto a los diferentes aspectos de la experiencia formativa? ¿Cuáles son sus motivaciones y sus expectativas respecto a los estudios?

Específicamente, el estudio indaga y presenta resultados vinculados a los siguientes objetivos:

- Conocer las motivaciones y las expectativas del estudiantado en el acceso al máster oficial en Educación.

- Analizar la satisfacción y la integración a los estudios de máster.

- Valorar las expectativas y los proyectos de futuro de los estudiantes de másteres.

\section{Método}

En este trabajo de investigación se utilizó una metodología cuantitativa de carácter descriptivo, basada en la aplicación de un cuestionario como técnica de recogida de información. La finalidad fue analizar las motivaciones y las expectativas en la decisión de cursar un máster, así como el proceso de adaptación posterior de tres promociones de estudiantes del ámbito de la educación.

\subsection{Participantes}

La población se definió como estudiantes de máster oficial del área de Educación de las universidades de Barcelona (UB) y Autónoma de Barcelona (UAB), procedentes de las siguientes titulaciones: másteres en Psicopedagogía, Intervención Social y Educativa, Educación para la Ciudadanía y Valores e Investigación en Didáctica, Formación y Evaluación Educativa (de la UB), y Soporte para la Inclusión Educativa y Social y Mediación: especialidad Mediación Comunitaria (de la UAB).

La muestra final que ha participado en el estudio es de 297 universitarios: 159 de los cuales proceden de la cohorte 2011-2012 y 138 de la cohorte 20122013. El 79\% son alumnos de la UB. La distribución por sexos confirma los datos para la población general del área de educación: el $80 \%$ son mujeres, frente al $20 \%$ de hombres. En cuanto a la edad, se corrobora la heterogeneidad del perfil —edades comprendidas en un intervalo de 20 a 50 años-, aunque el tanto por ciento mayoritario es para el perfil más joven. Se observa un aumento de estudiantes internacionales en comparación con el grado, sobre todo en másteres con un perfil más investigador. En la tabla 1 se especifican las características de los participantes en la investigación. 
Tabla 1. Características de la muestra participante en el estudio

\begin{tabular}{|c|c|c|c|c|c|c|}
\hline \multirow[t]{2}{*}{ Universidad } & \multirow[t]{2}{*}{ Máster } & \multicolumn{2}{|c|}{ Curso 2011-2012 } & \multicolumn{2}{|c|}{ Curso 2012-2013 } & \multirow{2}{*}{$\begin{array}{c}\text { Error } \\
\text { muestral }\end{array}$} \\
\hline & & Población & $\begin{array}{c}\text { Muestra } \\
\text { (\% de } \\
\text { participación) }\end{array}$ & Población & $\begin{array}{c}\text { Muestra } \\
(\% \text { de } \\
\text { participación) }\end{array}$ & \\
\hline \multirow{4}{*}{$\begin{array}{l}\text { Universidad } \\
\text { de } \\
\text { Barcelona }\end{array}$} & $\begin{array}{l}\text { Psicopeda- } \\
\text { gogía }\end{array}$ & 50 & 48 & 50 & 48 & \multirow{6}{*}{1,7} \\
\hline & $\begin{array}{l}\text { Intervención } \\
\text { Social y Edu- } \\
\text { cativa }\end{array}$ & 30 & 29 & 30 & 27 & \\
\hline & $\begin{array}{l}\text { Educación } \\
\text { para la Ciu- } \\
\text { dadanía y } \\
\text { Valores }\end{array}$ & 25 & 24 & 11 & 10 & \\
\hline & $\begin{array}{l}\text { Investigación } \\
\text { en Didáctica, } \\
\text { Formación y } \\
\text { Evaluación } \\
\text { Educativa }\end{array}$ & 25 & 24 & 18 & 16 & \\
\hline \multirow[t]{2}{*}{$\begin{array}{l}\text { Universidad } \\
\text { Autónoma } \\
\text { de } \\
\text { Barcelona }\end{array}$} & $\begin{array}{l}\text { Soporte para } \\
\text { la Inclusión } \\
\text { Educativa y } \\
\text { Social }\end{array}$ & 20 & 16 & 22 & 18 & \\
\hline & Mediación & 21 & 18 & 23 & 19 & \\
\hline
\end{tabular}

Fuente: elaboración propia.

\subsection{Instrumento de recogida de información}

La utilización del método por encuesta, tal y como expresan Torrado (2004) y Ruiz (2009), es de vital relevancia en el ámbito académico, debido a su utilidad para abordar fenómenos educativos y conseguir la aproximación a la realidad mediante la realización de preguntas sobre una gama de sucesos presentes, pasados o futuros.

Desde una perspectiva cuantitativa, se parte de datos estructurados y representativos que posibilitan conocer la transición a los estudios de máster, así como el proceso que viven los estudiantes durante el mismo. La encuesta en formato de cuestionario facilita la contextualización del fenómeno a examinar, así como su generalidad en el campo de análisis.

La investigación, como ya se ha adelantado, cuantitativa y de carácter descriptivo, se ayuda de la aplicación de una consulta en formato papel y en línea a partir del cuestionario "Análisis de los estudiantes de máster del área de ciencias sociales» (Llanes-Ordóñez et al., 2015). El instrumento incluyó un total de 27 ítems (ver la tabla 2). Las dimensiones de análisis han sido: 1) procedencia académica previa; 2) trayectoria laboral previa; 3) motivos de elección del máster; 4) proyecto profesional; 5) satisfacción académica; 6) factores favorecedores o inhibidores de la transición, y 7) transición académico-laboral. El cuestionario incluye ítems de formato diferente (de respues- 
Tabla 2. Especificidad del instrumento

\begin{tabular}{ll}
\hline \multicolumn{1}{c}{ Dimensiones de análisis } & \multicolumn{1}{c}{ Ítems } \\
\hline Datos de identificación & $\begin{array}{l}12 \text { preguntas con opciones de respuesta para conocer } \\
\text { datos sociodemográficos y técnicos del máster. }\end{array}$ \\
\hline Procedencia académica previa & 2 preguntas abiertas. \\
\hline Trayectoria laboral previa & 4 preguntas con opciones de respuesta. \\
\hline Motivos de elección del máster & $\begin{array}{l}2 \text { preguntas; } 1 \text { escala Likert con } 10 \text { ítems a valorar entre } \\
1 \text { (nada) y } 5 \text { (mucho), y } 1 \text { pregunta con } 4 \text { opciones de } \\
\text { respuesta. }\end{array}$ \\
\hline Proyecto profesional & $\begin{array}{l}1 \text { escala Likert con } 26 \text { ítems a valorar entre } 1 \text { (nada) y } 6 \\
\text { (mucho). }\end{array}$ \\
\hline Satisfacción académica & $\begin{array}{l}2 \text { preguntas; } 1 \text { escala Likert con } 8 \text { ítems a valorar entre } 1 \\
\text { (totalmente en desacuerdo) y } 5 \text { (totalmente de acuerdo), }\end{array}$ \\
\hline yactores favorecedores o inhibido- 1 pregunta con 2 opciones de respuesta. \\
res de la transición
\end{tabular}

Fuente: elaboración propia.

ta múltiple, escalas de valoración tipo Likert con cinco niveles de respuesta, diferencial semántico con siete niveles de respuesta y preguntas abiertas).

\subsection{Procedimiento}

Se contactó con los coordinadores de los másteres seleccionados (másteres del área de Educación de las dos universidades participantes: UB y UAB) para poder acceder al campo de investigación. Ello implicó identificar los másteres del área de Educación de las universidades participantes y seleccionarlos teniendo en cuenta su idiosincrasia - investigadores o profesionalizadores-. Una vez obtenida la información, se pasó a una matriz de datos que fueron objeto de análisis en función de los objetivos propuestos.

\subsection{Análisis de la información}

En el campo de las ciencias sociales es prácticamente inevitable, hoy en día, no realizar un análisis de la investigación educativa de datos cuantitativos planteado a través de procesos estadísticos mediante el programa informático SPSS-Win (Statistical Package for the Social Sciences) (Vila, 2006).

Con los datos se han realizado los siguientes análisis estadísticos: descriptivos univariante y multivariante, inferencial y bivariante.

La estadística descriptiva univariante se ha llevado a cabo para cada una de las variables respetando en todo momento la naturaleza de las mismas y se han hallado las tablas de frecuencias.

Se ha estudiado el ajuste de la distribución de las variables cuantitativas a la distribución de la Ley Normal para confirmar uno de los requisitos indispensables en la aplicación de pruebas paramétricas o no paramétricas dentro del con- 
junto de pruebas de significación. Se han utilizado dos métodos: por un lado, el intuitivo, a partir de las diferentes representaciones gráficas, y, por otro, la prueba específica de bondad de ajuste Kolmogorov-Smirnov, que compara la distribución empíricamente observada con la distribución normal teórica, con lo que detecta las diferencias entre ambas distribuciones (Torrado, 2012).

La estadística descriptiva bivariante o inferencial se ha utilizado para confeccionar, por un lado, tablas de contingencia comparando variables a partir de chi-cuadrado que ayudarán a mostrar significatividad o ausencia de la misma en el estudio. Y, por otro lado, análisis de prueba de contraste no paramétrica para más de dos muestras independientes a partir de la prueba ANOVA de un factor.

\section{Resultados}

Los resultados de este estudio se presentan agrupados en varias dimensiones relacionadas con los objetivos y las variables analizadas. Se han tenido en cuenta los siguientes ítems del cuestionario para los objetivos planteados: sexo, edad, nota media de acceso al máster, formación previa, tipología de acceso y situación laboral previa. Estos ítems han estado contrastados con la escala de motivos y expectativas en el acceso, la satisfacción con la experiencia académica y las expectativas de futuro profesional una vez terminada la formación. Las variables serán analizadas a partir de tres momentos clave del proceso (acceso al máster —inicio—, proceso de formación — durante - y proyecto de futuro - final-).

\subsection{Motivaciones en el acceso al máster (inicio)}

$\mathrm{Al}$ analizar la escala de los motivos que han llevado a los estudiantes a acceder al máster, las medias de los encuestados quedan representadas tal y como se ve en la figura 1. Los dos ítems mayoritarios han vinculado la decisión a cuestiones formativas (adquirir competencias profesionales e incrementar el bagaje formativo) y, en menor medida, acceder al doctorado o conseguir reconocimiento social. Ahora bien, cuando se les pregunta directamente por el motivo principal de acceso, señalan lo siguiente: en un $65,4 \%$, alcanzar una especialización profesional; en un 13,3\%, redefinir su proyecto profesional (cambio de carrera); en un $11 \%$, cambio de área dentro de la misma carrera profesional, y en un $10,3 \%$, promoción laboral.

Cuando se realiza el análisis en base a las variables planteadas por el estudio, hay que resaltar que no hay diferencias significativas en cuanto a la edad y al sexo. Por ello, los motivos son similares tanto para hombres como para mujeres, así como para los distintos grupos de edad (desde recién titulados hasta personas con más de 50 años). Tampoco encontramos desigualdades en la tipología. Sí que se han hallado diferencias en la variable situación laboral previa, lo que determina que el estar trabajando o no condiciona los motivos de acceso. En cuanto a la nota media, variable que en un primer momento 
podría parecer que condiciona los motivos de acceso, estadísticamente no presenta diferencias, y por tanto no se puede determinar que el rendimiento académico previo contribuya a explicar las motivaciones necesarias para realizar un máster.

Aquellos que trabajan a tiempo completo (ver la tabla 3) y, además, su empleo está relacionado con la formación, acceden al máster para ampliar competencias profesionales y adquirir un mejor bagaje formativo, mientras que aquellos que no trabajan persiguen mejorar su situación profesional $\left(F_{(2,1)}=2,9 ; p<0,05\right)$.

Asimismo, aunque no se han encontrado diferencias significativas entre graduados, diplomados y licenciados (ver la tabla 4), es de resaltar que aquellos

Figura 1. Motivos de acceso al máster

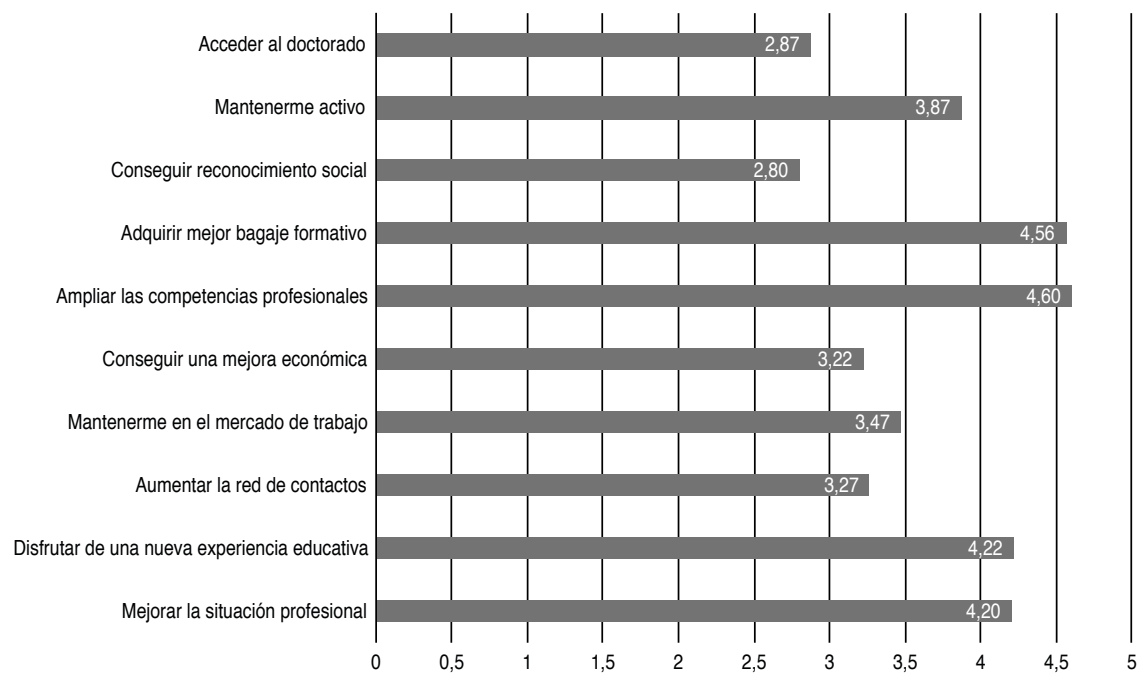

Fuente: elaboración propia.

Tabla 3. Relación de los motivos con la situación laboral en el momento de acceder

\begin{tabular}{lcccccccc}
\hline \multicolumn{1}{c}{ Motivos de acceso } & \multicolumn{2}{c}{$\begin{array}{c}\text { Trabajo a tiempo } \\
\text { completo }\end{array}$} & \multicolumn{2}{c}{ Paro } & gl & $\boldsymbol{F}$ & $\boldsymbol{p}$ \\
\cline { 2 - 6 } & $\boldsymbol{X}$ & $\boldsymbol{D} \boldsymbol{s}$ & $\boldsymbol{X}$ & $\boldsymbol{D} \mathbf{s}$ & & & \\
\hline $\begin{array}{l}\text { Adquirir competencias } \\
\text { profesionales }\end{array}$ & 4,69 & 0,61 & 4,51 & 0,58 & 2,1 & 3,66 & $<0,05$ \\
$\begin{array}{l}\text { Adquirir mejor bagaje } \\
\text { formativo }\end{array}$ & 4,67 & 0,61 & 4,56 & 0,67 & 2,1 & 3,66 & $<0,05$ \\
$\begin{array}{l}\text { Mejorar la situación } \\
\text { profesional }\end{array}$ & 4,13 & 0,98 & 4,34 & 0,84 & 2,1 & 3,85 & $<0,05$ \\
\hline
\end{tabular}

$X=$ media; $D s=$ desviación típica; $g l=$ grados de libertad; $F=$ frecuencia; $p=$ probabilidad

Fuente: elaboración propia. 
Tabla 4. Relación de los motivos con la formación previa

\begin{tabular}{lccccccc}
\hline \multirow{2}{*}{ Motivos de acceso } & \multicolumn{2}{c}{ Graduados } & \multicolumn{3}{c}{ Diplomados } & \multicolumn{2}{c}{ Licenciados } \\
\cline { 2 - 7 } & $\boldsymbol{X}$ & $\boldsymbol{D}$ & $\boldsymbol{X}$ & $\boldsymbol{D s}$ & $\boldsymbol{X}$ & $\boldsymbol{D} \mathbf{s}$ \\
\hline Mejorar la situación profesional & 4,50 & 0,54 & 4,10 & 0,97 & 4,34 & 0,84 \\
Ampliar competencias profesionales & 4,83 & 0,40 & 4,60 & 0,62 & 4,60 & 0,66 \\
Adquirir un mejor bagaje formativo & 4,67 & 0,51 & 4,56 & 0,70 & 4,59 & 0,69 \\
\hline Conseguir reconocimiento social & 2,50 & 1,04 & 2,74 & 1,08 & 2,87 & 1,12 \\
\hline
\end{tabular}

$X=$ media; $D s=$ desviación típica

Fuente: elaboración propia.

que acaban de finalizar estudios de primer ciclo continúan la formación, en gran medida para conseguir mejorar su situación profesional sin obviar la parte formativa. A su vez, aquellos que llevan años fuera del sistema educativo (diplomados o licenciados) vuelven a la formación para centrar la mirada en el aprendizaje: adquirir un mejor bagaje formativo y competencias profesionales. Los tres grupos coinciden en resaltar que lo que menos les interesa es el reconocimiento social.

\subsection{Integración y satisfacción en el máster (durante los estudios)}

La escala de satisfacción académica de los encuestados destaca la relación con los compañeros como el ítem más positivo en su satisfacción respecto a los estudios, seguido de la satisfacción con la decisión y el ambiente educativo que propicia el máster. El ítem peor valorado está vinculado a la estimulación académica que hay en clase. Son críticos con el clima del aula desde la óptica de la calidad formativa.

El análisis comparativo de la escala con la edad, el sexo, la nota media, la tipología en el acceso y la situación laboral previa no presenta diferencias significativas. Ahora bien, aquellos que acceden a partir de un postgrado u otro máster presentan un nivel mayor de satisfacción general con los nuevos estudios que aquellos que transitan a partir de una diplomatura (ver tabla 5) y, por tanto, le dan un mayor valor al máster aquellos que ya han realizado estudios de segundo ciclo $\left(F_{(3,1)}=1,4 ; p<0,05\right)$.

En cuanto a la integración en los estudios, a partir del diferencial semántico de los factores de transición, la tipología de acceso presenta diferencias

Tabla 5. Satisfacción académica en relación con la formación de acceso

\begin{tabular}{lccccccc}
\hline & \multicolumn{2}{c}{$\begin{array}{c}\text { Posgrado o } \\
\text { máster }\end{array}$} & Diplomatura & gl & $\boldsymbol{F}$ & $\boldsymbol{p}$ \\
\cline { 2 - 6 } & $\boldsymbol{X}$ & $\boldsymbol{D} \boldsymbol{s}$ & $\boldsymbol{X}$ & $\boldsymbol{D} \boldsymbol{s}$ & & & \\
\hline $\begin{array}{l}\text { Satisfacción académica con los } \\
\text { nuevos estudios }\end{array}$ & 4,36 & 0,65 & 3,96 & 0,90 & 3,1 & 4,13 & 0,05 \\
\hline
\end{tabular}

$X=$ media; $D s=$ desviación típica; $g l=$ grados de libertad; $F=$ frecuencia; $p=$ probabilidad

Fuente: elaboración propia. 
Figura 2. Satisfacción con la experiencia académica $(n=297)$

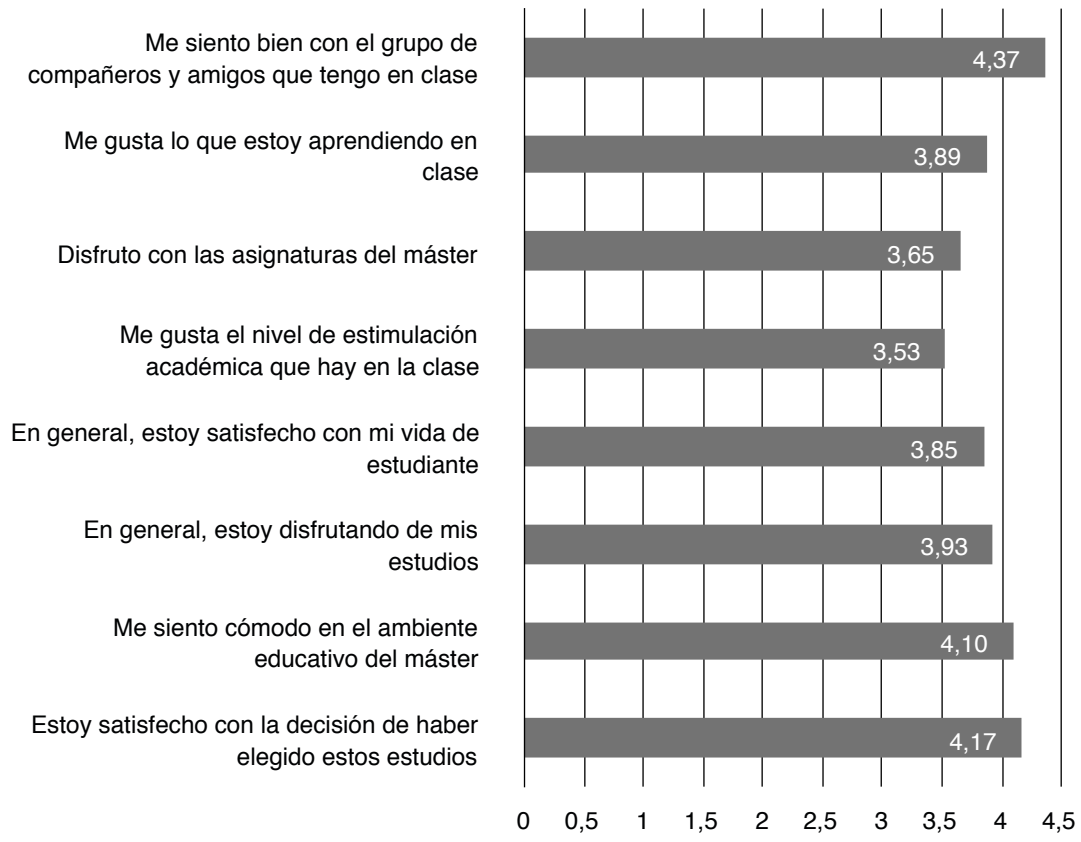

Fuente: elaboración propia.

significativas atendiendo a la prueba ANOVA de un factor. En términos generales, las personas que han acabado estudios de grado hace más de un año y que no tienen experiencia profesional relacionada con la formación otorgan una media alta a todos los ítems asociados a esta nueva experiencia educativa.

Ahora bien, la tipología de acceso presenta diferencias significativas para cuatro de los factores de transición académica: adecuación de los estudios a sus intereses, problemas familiares durante la formación, interés del contenido de las asignaturas y soporte personal a lo largo de los estudios (ver tabla 6).

La aplicación de la prueba Scheffé $\left(F_{(3,01)}=6,91 ; p<0,05\right)$ establece diferencias significativas en la comparación de medias, determinando que los recién titulados que transitan al máster directamente o aquellos que llevan un año en el mercado de trabajo tras acabar la formación del primer ciclo y no tienen experiencia laboral son los que mayor apoyo han tenido por parte de la familia y su entorno próximo para acceder a la formación, así como durante su realización. La prueba Duncan $\left(F_{(3,01)}=4,69 ; p<0,05\right)$ concreta que los profesionales con experiencia sénior y los estudiantes que acceden tras un año en el mercado de trabajo y no tienen experiencia profesional son los que otorgan una mayor media al interés del contenido del máster, valorando por tanto positivamente la formación. La prueba HSD de 
Figura 3. Tipología de acceso al máster en relación con los factores de transición académica $(n=297)$

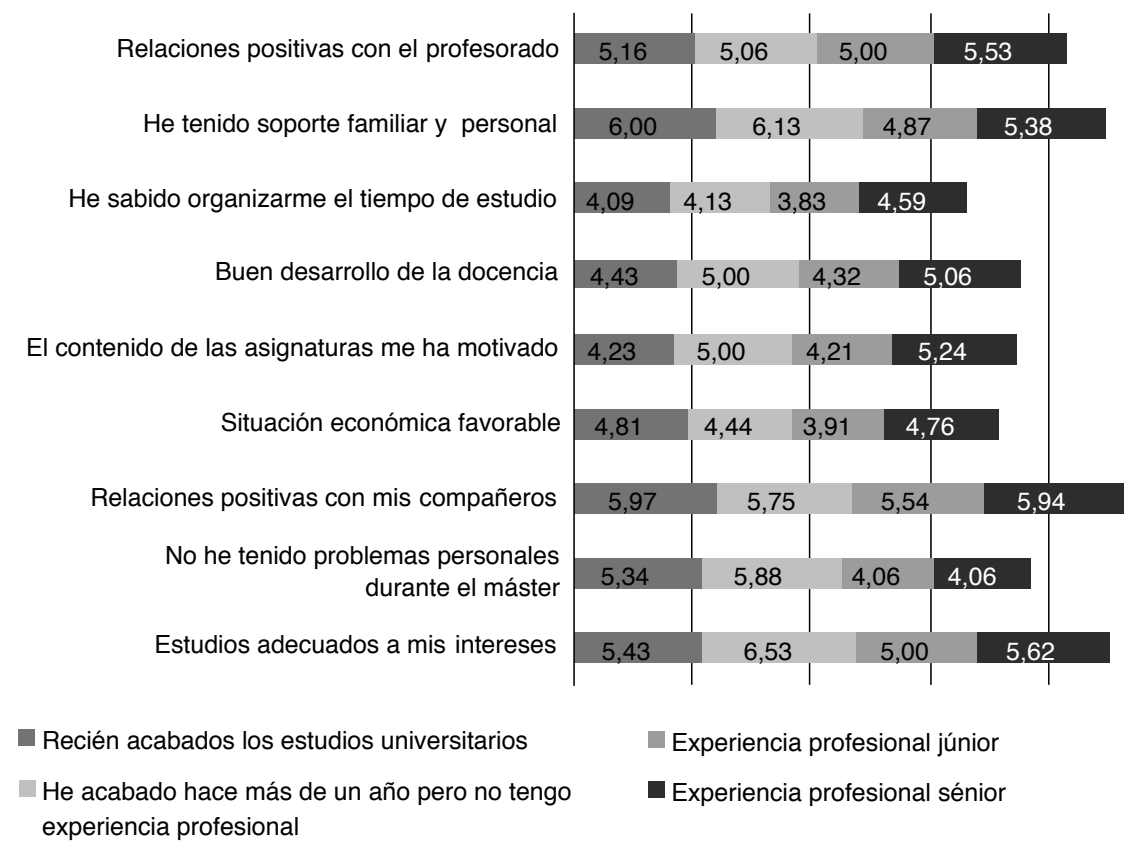

Fuente: elaboración propia.

Tukey $\left(F_{(3,01)}=6,08 ; p<0,05\right)$ especifica que los profesionales sénior y júnior son los que mayores cargas familiares tienen. Esta afirmación se relaciona con la edad de los participantes (en estos casos, por tónica general, de mayor edad) y la preocupación por los ascendentes está latente, así como por los descendentes. La prueba Duncan $\left(F_{(3,01)}=4,7 ; p<0,05\right)$ perfila que aquellos que acceden tras acabar la formación de primer ciclo directamente o los que llevan un año en el mercado de trabajo sin experiencia profesional resaltan que los estudios se adecuan a sus intereses. En este sentido, los que tienen experiencia profesional son más exigentes con la adecuación del máster a su campo de especialización. 
Tabla 6. Tipología de acceso al máster y factores de transición académica

\begin{tabular}{|c|c|c|c|c|c|c|c|c|c|c|c|}
\hline & \multicolumn{2}{|c|}{$\begin{array}{l}\text { Recién } \\
\text { titulado de } \\
\text { grado }\end{array}$} & \multicolumn{2}{|c|}{$\begin{array}{l}\text { Más de un } \\
\text { año tras } \\
\text { finalizar } \\
\text { el grado } \\
\text { pero sin } \\
\text { experiencia }\end{array}$} & \multicolumn{2}{|c|}{$\begin{array}{l}\text { Profesional } \\
\text { júnior }\end{array}$} & \multicolumn{2}{|c|}{$\begin{array}{l}\text { Profesional } \\
\text { sénior }\end{array}$} & \multirow[t]{2}{*}{$g l$} & \multirow[t]{2}{*}{$\boldsymbol{F}$} & \multirow[t]{2}{*}{$p$} \\
\hline & $x$ & $D s$ & $x$ & Ds & $x$ & $D s$ & $x$ & $D s$ & & & \\
\hline $\begin{array}{l}\text { Estudios } \\
\text { adecuados } \\
\text { a mis inte- } \\
\text { reses }\end{array}$ & 5,43 & 1,50 & 6,53 & 0,64 & 5,00 & 1,62 & 5,02 & 1,59 & 3,01 & 5,47 & 0,05 \\
\hline $\begin{array}{l}\text { No he } \\
\text { tenido } \\
\text { problemas } \\
\text { personales } \\
\text { durante el } \\
\text { máster }\end{array}$ & 5,34 & 1,86 & 5,87 & 1,14 & 4,06 & 1,97 & 4,06 & 1,13 & 3,01 & 4,56 & 0,05 \\
\hline $\begin{array}{l}\text { El conteni- } \\
\text { do de las } \\
\text { asignaturas } \\
\text { me ha } \\
\text { motivado }\end{array}$ & 4,23 & 1,58 & 5,00 & 1,09 & 4,21 & 1,74 & 5,14 & 1,57 & 3,01 & 4,66 & $<0,05$ \\
\hline $\begin{array}{l}\text { He tenido } \\
\text { soporte } \\
\text { familiar y } \\
\text { personal }\end{array}$ & 6,00 & 1,52 & 6,13 & 1,08 & 4,87 & 1,70 & 5,38 & 1,70 & 3,01 & 5,44 & $<0,05$ \\
\hline
\end{tabular}

$X=$ media; $D s$ = desviación típica; $g l=$ grados de libertad; $F=$ frecuencia; $p=$ probabilidad

Fuente: elaboración propia.

\subsection{Proyección profesional (final del proceso)}

Aunque la mayoría de los encuestados señala que ven una oportunidad de inserción profesional a partir del máster, son aquellos que han acabado hace un año y no tienen experiencia profesional los que lo han explicitado en mayor medida. Además, en términos generales, estos son los que mejor valoran su paso por el máster para su futura proyección profesional $\left(F_{(2,21)}=2,74\right.$; $p<0,05)$. Aunque son optimistas, en el momento de especificar cómo será esta proyección no se sienten capacitados para explicarla.

A partir de las respuestas de los encuestados, se han hallado diferencias significativas entre sus expectativas y su proyección futura tras finalizar la formación de máster. Aquellos que acceden al máster con la idea de continuar estudios de doctorado resaltan las cargas familiares como un factor negativo a la hora de continuar con la formación de tercer ciclo $\left(F_{(2,21)}=7,18\right.$; $p<0,05)$. Sin embargo, este es el menor de los problemas para aquellos que acceden a estudios de máster con la idea de encontrar trabajo relacionado con esta nueva formación. En cuanto a las relaciones con el profesorado $\left(F_{(2,21)}=\right.$ $4,67 ; p<0,05)$, los que tienen intención de acceder a estudios de doctorado 
especifican que disfrutan de mejores relaciones con el profesorado que el resto de estudiantes.

\section{Conclusiones}

En sintonía con el objetivo de esta formación de segundo ciclo y atendiendo a la finalidad descrita por los descriptores de Dublín, los estudiantes encuestados han señalado que en el máster buscaban una especialización de su campo profesional, así como la adquisición de competencias laborales y un mayor bagaje formativo. Ahora bien, en el caso de los másteres del área de educación, hay un porcentaje de profesionales que acceden a la formación para redefinir su proyecto profesional (un 13,3\%) o cambiar de área de trabajo dentro de la misma profesión (un 11\%). Por tanto, además de ser un espacio de especialización, se trata de un entorno que permite hacer un cambio de carrera (Pegalajar, 2016; Serrano y Pontes, 2015).

En coherencia con otros estudios previos (Llanes-Ordóñez et al., 2015), el análisis permite identificar cuatro perfiles o tipologías de estudiantes de máster:

1. Personas que acceden a la formación de segundo ciclo directamente desde el primer ciclo.

2. Personas que acceden a la formación de segundo ciclo tras finalizar la formación de primer ciclo y haber pasado por el mercado laboral, pero no han conseguido trabajar en su ámbito formativo.

3. Experiencia profesional júnior, es decir, con menos de cinco años de experiencia laboral.

4. Experiencia profesional sénior, es decir, con igual o más de cinco años de experiencia laboral.

En este sentido, tal y como se manifiesta en Savickas (2012) y Savickas et al. (2009), los recién graduados continúan la formación por falta de expectativas profesionales, intentando estar mejor preparados para enfrentarse al mercado laboral.

En consonancia con la literatura existente (Rienties et al., 2014; Romero y Figuera, 2016), los másteres acogen a un perfil de alumnos mucho más heterogéneo que la formación de primer ciclo. En este caso se trata de estudiantes extranjeros, intereses profesionales, formación previa realizada o experiencia laboral. Por tanto, la adecuación a partir de programas de orientación y tutoría serán fundamentales para contribuir a la empleabilidad de sus estudiantes.

El análisis de la satisfacción académica en función de las tipologías de los alumnos ha mostrado diferencias a tener en cuenta: aquellos que ya han vivido experiencias educativas de segundo ciclo valoran con mayor grado de satisfacción la nueva formación que aquellos que finalizaron la carrera hace años y vuelven a la formación buscando una nueva experiencia educativa. Además, aquellos que tienen experiencia laboral son los que conectan de manera más directa el sentido del contenido del máster con el ejercicio profesional, resal- 
tando el interés práctico de la formación. En contraposición, los recién graduados o aquellos que no tienen experiencia laboral relacionada con el campo de estudio no son capaces de entender o ver este valor. En ocasiones, la falta de una perspectiva profesional clara les imposibilita ver la importancia formativa del máster desde esa óptica profesional (Figuera et al., 2018).

Aun considerando las casuísticas expresadas, en términos generales los encuestados señalan la formación de segundo ciclo como una oportunidad, aunque esta adquiera matices dependiendo de la formación previa, la trayectoria laboral o el motivo determinante para el acceso. En términos generales, la satisfacción académica con el máster es alta.

Este análisis nos aproxima a la realidad de los estudiantes de másteres del área de educación en dos universidades públicas del territorio catalán, aunque hay que continuar realizando investigaciones para conocer las motivaciones y las expectativas del alumno (incremento de universidades y de comunidades autónomas) para tener una visión global de la realidad de la formación de segundo ciclo en España. Damos importancia a esta línea de estudios, ya que la formación de segundo ciclo ha resultado imprescindible desde tres vertientes: especialización, acceso al doctorado o entrada en el ejercicio profesional para salidas laborales concretas. Quedan preguntas en el aire que hacen imprescindible el análisis de esta área para su conocimiento. Entre otras, serían: ¿Cómo intervienen las prácticas de máster en la mejora de competencias y en la inserción profesional? ¿Qué sentido debería tener el trabajo final de máster desde la perspectiva de la gestión de la carrera? ¿Cómo planificar una formación adecuada a las diferentes tipologías de estudiantes que acceden a una formación de segundo ciclo? También se deberían establecer procesos de triangulación de informantes que permitieran realizar una mayor aproximación a la realidad sobre el objeto de estudio, así como profundizar en las expectativas de futuro laboral y en el proyecto profesional de los estudiantes que se inicia durante la formación de máster. Dimensión que se analiza descriptivamente en esta investigación y que podría ser objeto de mayor profundización.

\section{Referencias bibliográficas}

AQU (2015). La inserció laboral dels titulats de màster. Recuperado de <http://www. aqu.cat/doc/doc_22262764_1.pdf>.

Armenteros, J. y Pérez, J. (2015). La Universidad Española en cifras 2013-2014. Madrid: CRUE.

Barry, M. y Mathies, B. (2011). An Examination of Master's Student Retention \& Completion. Comunicación presentada en el Association of Institutional Research Annual Forum. Toronto, Ontario.

Cabrera, A.F.; Pérez, P. y López, L. (2014). Evolución de las perspectivas de estudio de la retención universitaria en los EEUU: Bases conceptuales y puntos de inflexión. En P. Figuera (ed.). Persistir con éxito en la universidad: De la investigación a la acción (pp. 15-40). Barcelona: Laertes.

Conen, K.E. (2012). Persistence of master's students in the United States: Development and testing of a conceptual model. Comunicación presentada en el Annual meeting 
of the Association for the Study of Higher Education, Las Vegas, NV, 15-17. Recuperado de $<$ http://search.proquest.com/docview/1220694051>.

CounCil of THE European Union (2011). Informe EUCO. Recuperado de <https:// www.consilium.europa.eu/uedocs/cms_data/docs/pressdata/en/ec/119175.pdf>.

Egido-Gálvez, I.; Fernández-Díaz, M.J. y Galán, A. (2014). La dimensión social del proceso de Bolonia: Apoyos y servicios para grupos de estudiantes poco representados en las universidades españolas. Educación XX1, 17(2), 57-81.

Espacio Europeo De Enseñanza Superior (1999). Declaración de Bolonia. Recuperado de <http://eees.umh.es/contenidos/Documentos/DeclaracionBolonia.pdf>.

Figuera, P.; Buxarrais, M.; Llanes, J. y Venceslao, M. (2018). Perfil, motivación y satisfacción académica en los estudiantes de máster: El caso de Ciencias Sociales y Jurídicas. Estudios sobre Educación, 34, 219-237. $<$ http://dx.doi.org/10.15581/004.34.219-237>

Hauschildt, K.; Gwosć, C.; Netz, N. y Mishra, S. (2015). Social and Economic Conditions of Student Life in Europe: Synopsis of Indicators. Eurostudent V 20122015. Bielefeld: W. Bertelsmann Verlag.

Hernández, M.J. y Carrasco, V. (2012). Percepciones de los estudiantes del máster en formación del profesorado de educación secundaria: Fortalezas y debilidades del nuevo modelo formativo. Enseñanza \& Teaching, 30(2), 127-152.

Joint Quality Initiative, JQI (2004). Shared "Dublin» descriptors for Short Cycle, First Cycle, Second Cycle and Third Cycle Awards. Dublin: informal group. Recuperado de $<$ https://www.uni-due.de/imperia/md/content/bologna/dublin_descriptors.pdf>.

Llanes-Ordóñez, J.; Figuera-Gazo, P.; Jurado-De los Santos, P.; RomeroRodríguez, S. y Torrado-FonseCa, M. (2015). La transición a los estudios de máster en el ámbito de la educación: El caso de la universidad española. En T. RaItZ y P. Figuera (orgs.). Transiçôes dos estudantes: Reflexōes iberoamericanas (pp. 67-85). Curitiba, Brasil: CRV.

Manso, J. y Martín, J. (2014). Valoración del máster de formación de profesorado de educación secundaria: Estudio de casos en dos universidades. Revista de Educación, 364, 145-169.

MECD (2015). Datos y cifras del sistema universitario español: Curso 2014-2015. Recuperado de <https://www.educacionyfp.gob.es/dms/mecd/servicios-al-ciudadanomecd/estadisticas/educacion/universitaria/datos-cifras/Datos-y-Cifras-del-SUECurso-2014-2015.pdf>.

OCDE (2015). Panorama de la educación: Indicadores de la OCDE. Recuperado de $<$ http://www.mecd.gob.es/dctm/inee/internacional/panorama-de-la-educacion2015.-informe-espanol.pdf?documentId=0901e72b81ee9fa3 $>$.

Pegalajar, M.C. (2016). Evaluación del Proceso de Convergencia Europea desde la Perspectiva de Estudiantes del Máster de Secundaria. Revista Iberoamericana de Evaluación Educativa, 9(2), 65-80.

PIAAC (2013). Program for the International Assessment of Adult Competencies. Recuperado de <http://nces.ed.gov/surveys/piaac/>.

Ramírez, E. (2014). «¿Qué Estoy Haciendo Aquí? (What Am I Doing Here?)»: Chicanos/Latinos(as) Navigating Challenges and Inequalities During Their First Year of Graduate School. Equity \& Excellence in Education, 47(2), 167-186.

Rapp, K. y Golde, C. (2008). Transitions in context: An overview of U.S. graduate education. En K.A. ToKUno (ed.). Graduate students in transition: Assisting students through the first year (monográfico n. ${ }^{\circ}$ 50, pp. 9-26). Columbia, SC: University of South Carolina. 
Real Decreto 1393/2007, de 29 de octubre, por el que se establece la ordenación de las enseñanzas universitarias oficiales. Boletín Oficial del Estado, 30 de octubre de 2007, núm. 260.

Real Decreto 420/2015, de 29 de mayo, de creación, reconocimiento, autorización y acreditación de universidades y centros universitarios. Boletín Oficial del Estado, 17 de junio de 2015, núm. 144, pp. 50365 a 50380.

Rienties, B.; Luchoomun, D. y Tempelaar, D. (2014). Academic and social integration of Master students: A cross-institutional comparison between Dutch and international students. Innovations in Education and Teaching International, 51(2), 130-141.

Romero, S. y Figuera, P. (2016). La orientación en la Universidad. En A. ManzaNARES (ed.). Orientación a lo largo de la vida. Madrid: Wolters Kluver.

Ruiz, A. (2009). Método de encuesta: construcción de cuestionarios, pautas y sugerencias. Revista d'Innovació $i$ Recerca en Educació, 2(2), 96-110.

Serrano, R. y Pontes, A. (2015). Expectativas ante la formación inicial entre el alumnado de secundaria del máster de Profesorado de Enseñanza Secundaria. Revista de Investigación Educativa, 33(2), 489-505.

Sollemetzidis, I.; Bennett, P. y Leman, J. (2014). The Postgraduate Thaught Experience Survey. 2014. Hesligton York: The Higher Education Academic.

Torrado, M. (2004). Estudios de encuesta. En R. Bisquerra (ed.). Metodología de la investigación educativa (pp. 231-257). Madrid: La Muralla.

- (2012). El fenómeno del abandono en la Universidad de Barcelona: El caso de ciencias experimentales. Tesis doctoral. Universidad de Barcelona. Facultad de Pedagogía.

Savickas, M.L. (2012). Life Design: A Paradigm for Career Intervention in the 21st Century. Journal of Counseling \& Development, 90(1), 13-19.

Savickas, M.L.; Nota, L.; Rossier, J.; Dauwalder, J.P.; Duarte, M.; Guisard, J. y VAN Vianen, A. (2009). Life designing: A paradigm for career construction in the 21st century. Journal of Vocational Behavior, 75, 235-250.

VILA, R. (2006). ¿Cómo hacer un análisis cuantitativo de datos de tipo descriptivo con el paquete estadístico SPSS? Butlletí La Recerca, ficha 6. Recuperado de $<$ http://www.ub.edu/ice/recerca/pdf/ficha6-cast.pdf>. 